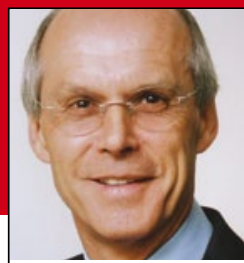

Prof. Dr. med. H. S. FüjeßI Isar-AmperKlinikum, KI. München-Ost, Haar

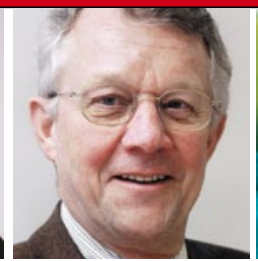

Prof. Dr. med. H. Holzgreve Internist, Kardiologische Praxis, München

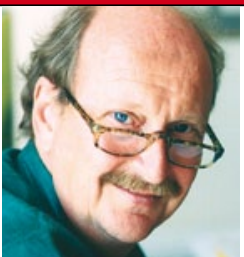

Prof. Dr. med. E. Ernst Peninsular Medical School, University of Exeter/UK

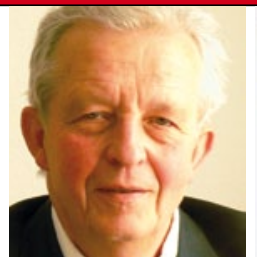

Prof. Dr. med. K. Malberg Immunologie, DresdenLoschwitz

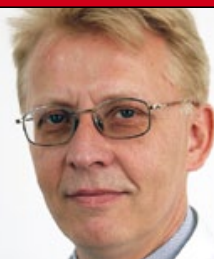

Prof. Dr. med.

K. Rasche

HELIOS Klinikum Wuppertal

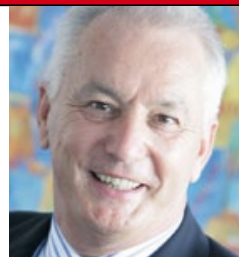

Prof. Dr. med. C. Diehm

SRH Klinikum Karlsbad

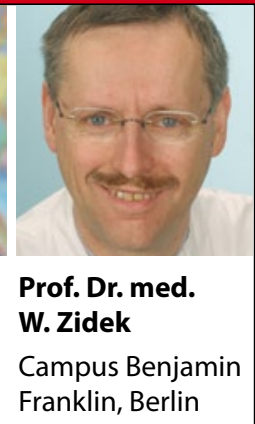

Campus Benjamin
Franklin, Berlin

\title{
Gebrechliche Senioren mit hohem Blutdruck leben länger
}

Auch im Alter sollte eine Hypertonie in der Regel behandelt werden, doch wer als Betagter Probleme mit dem Gehen hat, für den ist hoher Blutdruck quoad vitam ein Vorteil.

- Im Rahmen der NHANES-Studie wurden 2340 Personen jenseits des 65. Lebensjahres, die zu Hause wohnten, befragt und untersucht. Alle Probanden wurden aufgefordert, über eine Strecke von $6 \mathrm{~m}$ in gewohntem Tempo zu gehen. Das Ergebnis wurde als „schnell“ ( $\geq$ $0,8 \mathrm{~m} / \mathrm{s} ; \mathrm{n}=1307))$, „langsam“ $(<0,8$ $\mathrm{m} / \mathrm{s} ; \mathrm{n}=790)$ ) oder „unvollständig“ ( $\mathrm{n}=$ 243) bewertet, wenn der Test aus körperlichen oder Sicherheitsgründen nicht beendet werden konnte.

Innerhalb von im Mittel sechs Jahren verstarben 589 Personen. Bei der Auswertung wurden ein breites Spektrum von demografischen und sozialen Einflussfaktoren, Risikofaktoren, Begleiterkrankungen und eine eventuelle antihypertensive Therapie berücksichtigt. Hoher systolischer Blutdruck steigert bei Senioren mit zügigem Gehtest die Mortalität um 35\%, während er bei unvollständigem Gehtest die Mortalität erheblich um 63\% senkt (jeweils signifikant; s. Tab.). Bei hohem diastolischem Blut-

\section{Tabelle}

\section{Korrelation zwischen Blutdruck, Gehleistung und Mortalität im Alter}

\begin{tabular}{l|l|l|l} 
Gehleistung & $\begin{array}{c}\text { Schnell }(\geq 0,8 \mathrm{~m} / \mathrm{sec}) \\
\text { Mortalität }\end{array}$ & langsam $(<0,8 \mathrm{~m} / \mathrm{sec})$ & unvollständig \\
\hline Syst. $\geq \mathbf{1 4 0}$ vs. $<\mathbf{1 4 0}$ & $+35 \%(\mathrm{~s})$ & $+12 \%(\mathrm{~ns})$ & $-62 \%(\mathrm{~s})$ \\
\hline Diast. $\geq \mathbf{9 0}$ vs. $<\mathbf{9 0}$ & $-6 \%$ (ns) & $-25 \%(\mathrm{~ns})$ & $-90 \%(\mathrm{~s})$
\end{tabular}

druck ist die Mortalität niedriger, und zwar umso ausgeprägter, je schlechter die Gehleistung ist. Bei hinfälligen Personen erreicht die Reduktion sogar ca. 90\% (signifikant).

\section{Kommentar}

Die großen Interventionsstudien bei Altershypertonie haben zunächst bei Patienten jenseits des 65., später auch jenseits des 80 . Lebensjahres eindrucksvoll gezeigt, dass eine antihypertensive Therapie die Mortalität und Morbidität senkt. Häufig wird aber übersehen, dass in diese Studien die fitten und rüstigen Alten eingeschlossen wurden. Deshalb soll die medikamentöse Therapie nach den Empfehlungen der Fachgesellschaften auch auf Senioren in guter körperlicher und geistiger Verfassung beschränkt werden.
Grundsätzlich vergrößern sich mit dem Alter die biologischen Unterschiede und damit auch die Bewertung von Vitalparametern. Im Alter kann normaler Blutdruck schlecht sein, schlecht ist ein Blutdruckabfall. Normaler Blutdruck ist ein Zeichen von Gebrechlichkeit, Hypertonie muss kein Risiko, sondern kann ein Hinweis auf Vitalität und Rüstigkeit sein. Das gilt gleichermaßen für den systolischen und diastolischen Blutdruck. Der Bedarf an individueller Therapie nimmt mit dem Alter zu.

H. HOLZGREVE = 